\title{
Expansion Thoracoplasty Affects Lung Growth and Morphology in a Rabbit Model
}

\section{A Pilot Study}

\author{
J. Casey Olson MS, Kyle C. Kurek MD, \\ Hemal P. Mehta MS, Matt L. Warman MD, \\ Brian D. Snyder MD, PhD
}

Published online: 22 March 2011

(c) The Author(s) 2011. This article is published with open access at Springerlink.com

\begin{abstract}
Background Thoracic insufficiency syndrome represents a novel form of postnatal restrictive respiratory disease occurring in children with early-onset scoliosis and chest wall anomalies. Expansion thoracoplasty improves lung volumes in children with thoracic insufficiency syndrome; however, how it affects lung development is unknown.

Questions/purposes Using a rabbit model of thoracic insufficiency syndrome, we evaluated the effect of expansion thoracoplasty on the response of biologic mechanisms in the alveolar microstructure.

Methods Using archived material from a previous experiment, 10 4-week-old New Zealand rabbits were divided into three groups: normal $(n=3)$, disease $(n=3)$, and treated $(n=4)$. Left ribs four to eight were tethered in

The institution of one or more of the authors (BDS) has received funding from the Scoliosis Research Society, the Chest Wall and Spine Deformity Study Group, Synthes Spine (West Chester, PA), and the National Institutes of Health (Grant Number 1R21AR057880$01)$.

Each author certifies that his or her institution approved the animal protocol for this investigation and that all investigations were conducted in conformity with ethical principles of research.

This work was performed at Beth Israel Deaconess Medical Center and Children's Hospital.
\end{abstract}

J. C. Olson, H. P. Mehta, B. D. Snyder

Center for Advanced Orthopaedic Studies, Beth Israel Deaconess

Medical Center, Boston, MA, USA

J. C. Olson, H. P. Mehta

Department of Biomedical Engineering, Boston University,

Boston, MA, USA

K. C. Kurek

Department of Pathology, Children's Hospital, Harvard Medical

School, Boston, MA, USA seven rabbits at age 5 weeks to induce hypoplasia of the left hemithorax (disease). At age 10 weeks, four of these rabbits were treated by expansion thoracoplasty (treated). At age 24 weeks, lungs were excised and processed. Alveolar density and parenchymal airspace were measured on histologic sections. Immunohistochemistry was performed for vascular endothelial growth factor receptor 2 (angiogenesis), KI-67 (cell proliferation), and RAM-11 (macrophages).

Results Alveolar walls were poorly perfused and airspace fraction was larger (emphysematous) in disease rabbits than normal or treated rabbits. Immunohistochemistry provided inconclusive evidence to support the concept that pulmonary hypoplasia is induced by thoracic insufficiency syndrome and controlled by expansion thoracoplasty.

Conclusions Treatment of thoracic insufficiency syndrome by expansion thoracoplasty may prevent emphysematous changes in the alveolar microstructure, thereby enhancing gas exchange.

Clinical Relevance Creating an animal model for thoracic insufficiency syndrome should provide insight into the effect of expansion thoracoplasty on lung development otherwise clinically unattainable.

M. L. Warman

Departments of Orthopaedics and Genetics, Children's Hospital, Harvard Medical School, Boston, MA, USA

B. D. Snyder $(\square)$

Department of Orthopaedic Surgery, Children's Hospital, Harvard Medical School, 300 Longwood Avenue, Boston, MA 02115, USA

e-mail: Brian.Snyder@childrens.harvard.edu;

brian.snyder@tch.harvard.edu 


\section{Introduction}

Thoracic insufficiency syndrome (TIS) is the inability of the thorax to support normal respiration and postnatal lung growth [3, 9]. It reflects a novel form of restrictive respiratory disease and postnatal pulmonary hypoplasia occurring in children with early-onset scoliosis and anomalies of the thorax. TIS presumably interferes with neonatal pulmonary alveolarization, a process that slows exponentially from birth until it is largely completed around 5 years of age [6]. A deficit in alveolarization during this period is thought to be irrecoverable; thus, urgent intervention may be required in young children to reduce long-term pulmonary risk [1]. Campbell et al. [2] pioneered expansion thoracoplasty for the treatment of TIS using a Vertical Expandable Prosthetic Titanium Rib (VEPTR $^{\mathrm{TM}}$; Synthes Inc, West Chester, PA) to lengthen the constricted hemithorax, simultaneously correcting the thoracic hypoplasia and associated scoliosis by distracting open the concavity of the spine and chest wall deformity. The rationale for this treatment was that in growing children, removing the chest wall constriction and lengthening the thorax would mechanically stretch the lung, thereby increasing lung volume and stimulating acinar development. Clinical studies in children with TIS treated with $\operatorname{VEPTR}^{\mathrm{TM}}[2,4,8]$ have demonstrated an increase in total lung volume and a reduction in scoliosis. However, it remains unknown how expansion thoracoplasty affects the growth and development of the lung in affected patients.

The fragile health of children afflicted with TIS makes it technically challenging to systematically evaluate the interdependence of growth of the spine, thorax, and lung in vivo. Therefore, we developed a rabbit model that exhibits some of the clinical features of TIS in a preliminary pilot study [14]; we created a constricted hemithorax that induced thoracic hypoplasia and scoliosis in growing rabbits by unilaterally tethering the ribs in 5 -week-old rabbits. Using this model in another pilot study [13], we evaluated how expansion thoracoplasty affected thoracic volume, lung volume, respiratory mechanics, alveolar morphology, and spine deformity during growth. The observations suggested expansion thoracoplasty did not substantially affect total lung volumes: the volume of the constricted hemithorax was increased, but the volume of the ipsilateral lung was not. Expansion thoracoplasty did improve alveolar histomorphometry (airspace fraction) and appeared to improve alveolar capillary perfusion, although we observed no increase in the total number of alveoli. From that pilot study, we inferred expansion thoracoplasty may improve pulmonary growth by modifying or preventing the abnormal alveolar structure caused by experimental TIS. Thus, although expansion thoracoplasty improves lung volumes in our model and in children with TIS, the mechanisms by which it affects lung development remain unclear.

To assess how expansion thoracoplasty affects biologic mechanisms, we explored (1) alveolar microstructure development (proliferating and macrophage cell fractions); and (2) capillary development and perfusion (vascular endothelial growth factor receptor 2 [VEGFR-2] immunohistochemistry and red blood cells in eosin-stained sections).

\section{Materials and Methods}

Because a rabbit model for TIS and its treatment by expansion thoracoplasty had not been previously established, our hospital's Institutional Animal Care and Use Committee limited the number of rabbits allowed to undergo the surgical procedures. We therefore examined archived pulmonary tissue from our previous rabbit model of TIS [13]; no new experiments were performed. Thus, by necessity, this is a pilot study; the sample size is small and the study is underpowered. Descriptions of the surgical procedures, methods for acquiring lung volumes, and preparing excised lung tissue have been published previously [13] and therefore are only briefly summarized here.

In our previous study, 10 4-week-old New Zealand rabbits were divided into three groups: normal $(n=3)$, disease $(n=3)$, and treated $(n=4)$. Three healthy rabbits served as a control (normal group). At age 5 weeks, hypoplasia of the left hemithorax was induced in seven rabbits by passing a subperiosteal figure-of-eight ligature (Ethibond $\mathbb{R}$; Ethicon Inc, Somerville, NJ) posteriorly around the fourth to eighth left ribs, tethering them together to create a constricted left hemithorax (Fig. 1). Subsequently, four of these seven rabbits were treated by expansion thoracoplasty (treated group) at age 10 weeks and three were left untreated (disease group). The fused

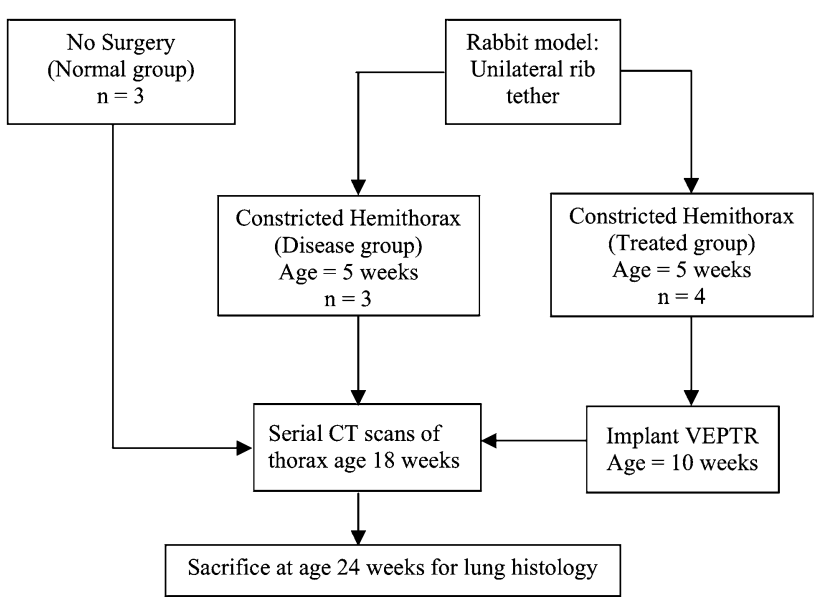

Fig. 1 A flow diagram shows an outline of the study. 
ribs were osteotomized at the apex of the concavity of the constricted left hemithorax using a dental burr. The thoracostomy was then distracted open using sublaminar hooks from a cervical spine instrumentation system (CerviFix $\AA$; Synthes Inc) to cradle the osteotomized ribs placed in opposition at either end of a 3.5-mm titanium rod (Fig. 2). At 24 weeks, all rabbits were ventilated on pure $\mathrm{O}_{2}$ for 10 minutes before euthanasia. The lungs and heart were excised and the lungs were infiltrated with formalin at $25 \mathrm{~mm} \mathrm{Hg}$ for 48 hours before being entirely embedded in paraffin. The paraffin-embedded lung samples were cut into $2-\times 2-\times 2-\mathrm{cm}$ tissue blocks selected by stratified random sampling [7] and were then cut into 7- $\mu \mathrm{m}$-thick tissue sections for histologic examination.

The rabbit is a useful model to understand postnatal pulmonary development in the human because rabbits have substantial postnatal alveolar development, which is thought to decrease at an exponential rate approaching maturity, similar to humans. Time points in this study were based on the normal timeline of skeletal maturation and alveolarization in the rabbit as compared with that of a human. The rabbit reaches skeletal maturity near 28 weeks of age, and although there may be some alveolarization potential through this time, the majority occurs before 16 weeks of age with approximately $25 \%$ of total alveoli present in the 5-week-old rabbit and $50 \%$ of total alveoli present in the 10-week-old rabbit [12]. Thus, after expansion thoracoplasty surgery, $50 \%$ of pulmonary growth potential remains in the growing rabbit. This physiological time point, measured by alveolar development remaining, is believed to be equivalent to approximately a 3-year-old child. The time point for euthanasia was chosen at a point in time when skeletal and pulmonary growths were both near completion.

In our previous study [13], alveolar number density (number of alveoli per unit of volume) was determined for the left and right lungs as described by Weibel and Gomez [21], and parenchymal airspace fraction was measured separately for each of the five lobes in rabbit lungs as described by Dunnill [7]. Morphometric analysis was performed by two independent reviewers (MJH, SRB) on hematoxylin and eosin-stained slides; interobserver variability was determined through regression and correlation (slope $=0.86$; correlation coefficient $=0.71$ ).

Immunohistochemistry (Table 1) was performed using antibodies against VEGFR-2, thyroid transcription factor 1 (TTF-1), macrophage-specific antigen (RAM-11), and KI-67 cell proliferation marker. Quantification was performed through cell fraction but only on macrophage and KI-67; VEGFR-2 stains were not localized enough to distinguish individually expressing cells and are displayed as a double stain with TTF-1 for insight on colocalization.

Previous morphologic analysis of excised lung tissue from our TIS rabbit model [13] demonstrated the left upper lobe (LUL) was modified the most, whereas the right lower lobe (RLL) was modified the least and could serve as an

Fig. 2A-C Three-dimensional CT reconstructions depict each experimental group: (A) a thoracic insufficiency syndrome (TIS) disease rabbit displaying fused ribs with a constricted left hemithorax; (B) a treated rabbit after expansion thoracoplasty of the left hemithorax; and (C) a normal healthy rabbit.
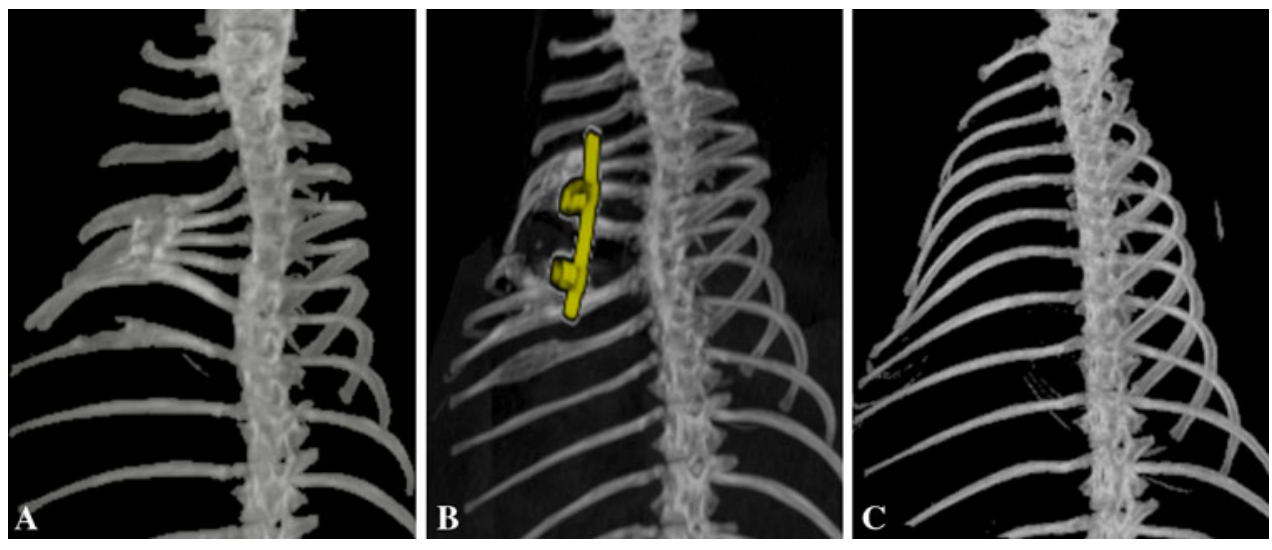

Table 1. Antibodies used in immunohistochemistry

\begin{tabular}{lllll}
\hline Antibody & Identifies & Company (catalog number) & $\begin{array}{l}\text { Primary concentration } \\
\text { (time) }\end{array}$ & Antigen retrieval \\
\hline VEGFR-2 & Angiogenesis signaling & $\begin{array}{l}\text { Santa Cruz Biotechnology Inc, } \\
\text { Santa Cruz, CA (6251) }\end{array}$ & $1: 200$ (1 hour) & Citrate at $125^{\circ} \mathrm{C}$ for 3 minutes \\
TTF-1 & Type II pneumocytes & DAKO, Carpinteria, CA (M3575) & $1: 150$ (1 hour) & Citrate at $125^{\circ} \mathrm{C}$ for 3 minutes \\
KI-67 & Proliferating cells & DAKO (M7240) & $1: 500$ (1 hour) & Citrate at $125^{\circ} \mathrm{C}$ for 3 minutes \\
RAM-11 & Macrophage cells & DAKO (M0633) & $1: 100$ (1 hour) & Citrate at $90^{\circ} \mathrm{C}$ for 40 minutes \\
\hline
\end{tabular}

VEGFR-2 = vascular endothelial growth factor receptor 2 ; TTF-1 = thyroid transcription factor 1 . 
internal control to understand the heterogeneity of biology within each group of animals. Therefore, only samples from the LUL and RLL of each animal were evaluated by immunohistochemistry. For each antibody stain in each rabbit, three sections with at least $100-\mu \mathrm{m}$ intervening distance between them were analyzed. The lung sections were deparaffinized, rehydrated, and then immersed in citrate solution, $\mathrm{pH} 6.0$, for epitope retrieval at either $90^{\circ} \mathrm{C}$ for 40 minutes or $125^{\circ} \mathrm{C}$ for 3 minutes in a pressure cooker. We visualized three single-stained sections with a peroxidase immunoenzyme labeling kit (Vectastain $\mathbb{R}$ ) Elite $\mathbb{R}$ ABC; Vector Laboratories Inc, Burlingame, CA) and developed in 3,3'-diaminobenzidine (DAB) substrate. Double-stained VEGFR-2/TTF-1 sections were stained sequentially using the peroxidase labeling kit developed with NovaRED ${ }^{\mathrm{TM}}$ substrate (Vector Labs) and then an alkaline phosphatase labeling kit (Vectastain (R ABC-AP kit; Vector Labs) with Vector B Blue substrate (Vector Labs). Crossreactivity between VEGFR-2 and TTF-1 antibodies (both mouse monoclonal) was inhibited using an additional mouse antibody blocking protocol between sequential stainings (Vector R M.O.M. ${ }^{\text {TM }}$ basic kit; Vector Labs). Endogenous peroxidase activity was blocked before the primary antibody application using a 3\% hydrogen peroxide solution for 10 minutes. All single stains were counterstained with hematoxylin and double stains had no counterstain.

For each of the stained tissue sections from each animal, five digital images were acquired at random by overlaying a grid of 1- $\times 1-\mathrm{mm}$ squares, numbered sequentially, and picking five numbers using a random number generator. The image at that location was then magnified $(\times 200)$. Cell counting was automated using a color deconvolution program [15] and MATLAB $\mathbb{R}$ functions in the Image Processing toolbox (The MathWorks Inc, Natick, MA). Immunohistochemistry signals for macrophages and KI-67positive cells were quantified in the alveolar space by counting the number of positively labeled cells normalized by the total number of cells with hematoxylin-stained nuclei. VEGFR-2/TTF-1 was not quantified in this manner because individual positively labeled cells could not be discerned; rather, they were examined for qualitative differences based on the intensity of the stain in the alveolar space.

Differences in alveolar cell counts of macrophages and KI-67-positive proliferating cells were determined between all experimental groups (normal, disease, and treated groups) using analysis of variance and Fisher's probable least significant difference by post hoc analysis. Qualitative observations were made of VEGFR-2/TTF-1 slides and hematoxylin and eosin-stained slides. We used Sigmaplot (Systat Software Inc, San Jose, CA) for all analyses.

\section{Results}

We could make no definite inference of whether expansion thoracoplasty influenced alveolar microstructure development based on a reduced number of macrophages (LUL: $\mathrm{F}=1.00, \mathrm{p}=0.43$; RLL: $\mathrm{F}=0.38, \mathrm{p}=0.70)(\mathrm{Fig} .3$ ) or
Fig. 3A-D Images illustrate the immunohistochemistry of lung tissue for antibody against RAM-11 (macrophage) in the three study groups: (A) disease lung, (B) normal lung, and (C) treated lung. RAM-11-positive cells are brown $\left(3,3^{\prime}\right.$-diaminobenzidine) and cell nuclei blue (hematoxylin) (original magnification, $\times 200$ ). (D) Positively stained cells were counted and are given as a percentage of total cell number. The left upper lobe (LUL) of disease rabbits had insignificantly higher cell counts. Sections from the right lower lobe (RLL) also show high variability. Error bars $=1 \mathrm{SD}$.
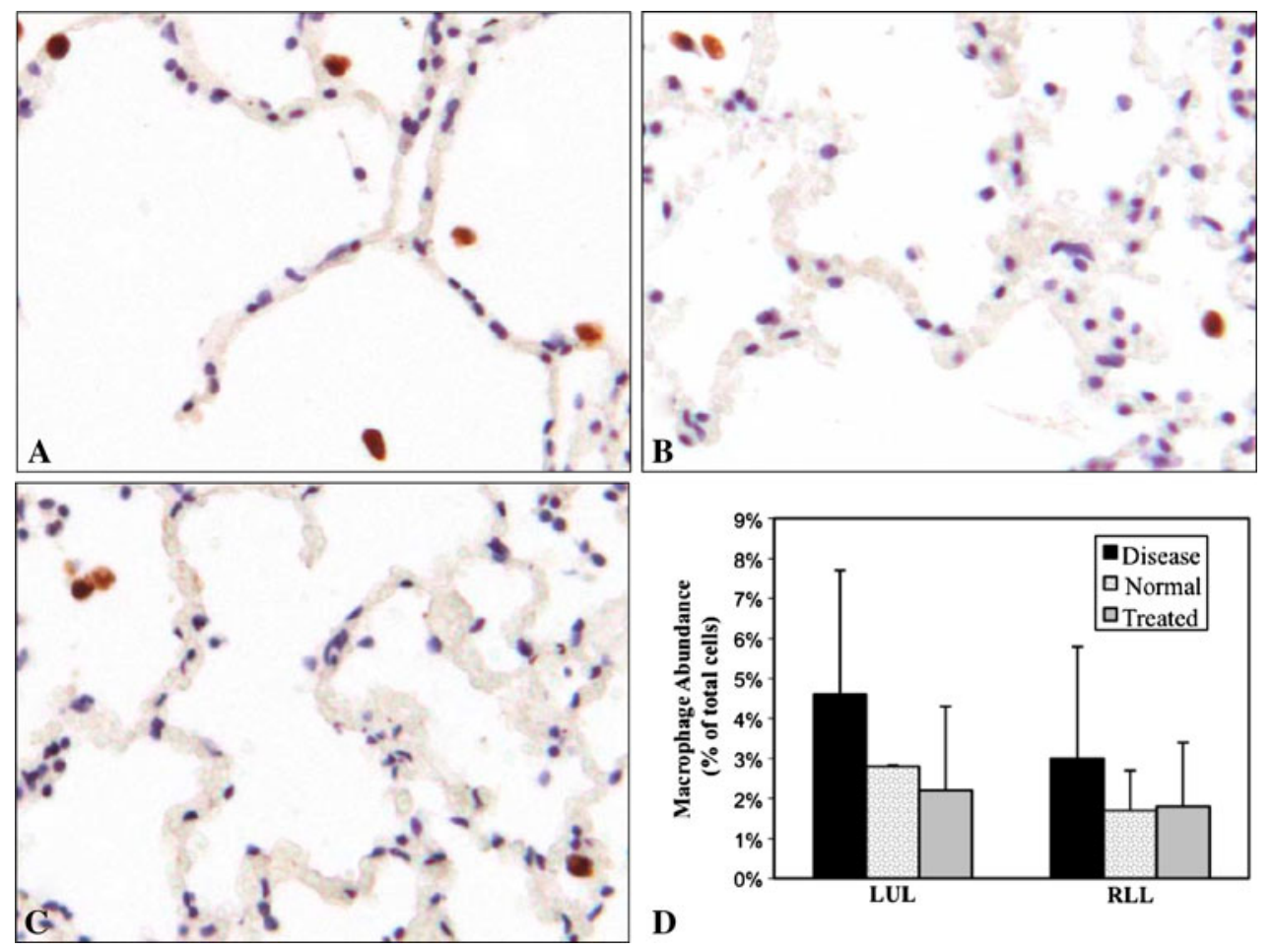
Fig. 4A-D Images illustrate the immunohistochemistry of lung tissue for KI-67 antibody (proliferation marker) in the three study groups: (A) disease lung, (B) normal lung, and (C) treated lung. KI-67-positive cells are brown (3,3'-diaminobenzidine) and cell nuclei blue (hematoxylin) (original magnification, $\times 200$ ). (D) Positively stained cells were counted and are given as a percentage of total cell number. The number of proliferating cells (KI-67-positive) was insignificantly decreased in the left upper lobe (LUL) of disease lungs compared with both treated and normal lungs. Similar numbers of KI-67-positive cells were observed among the groups for the right lower lobe (RLL). Error bars $=1 \mathrm{SD}$.

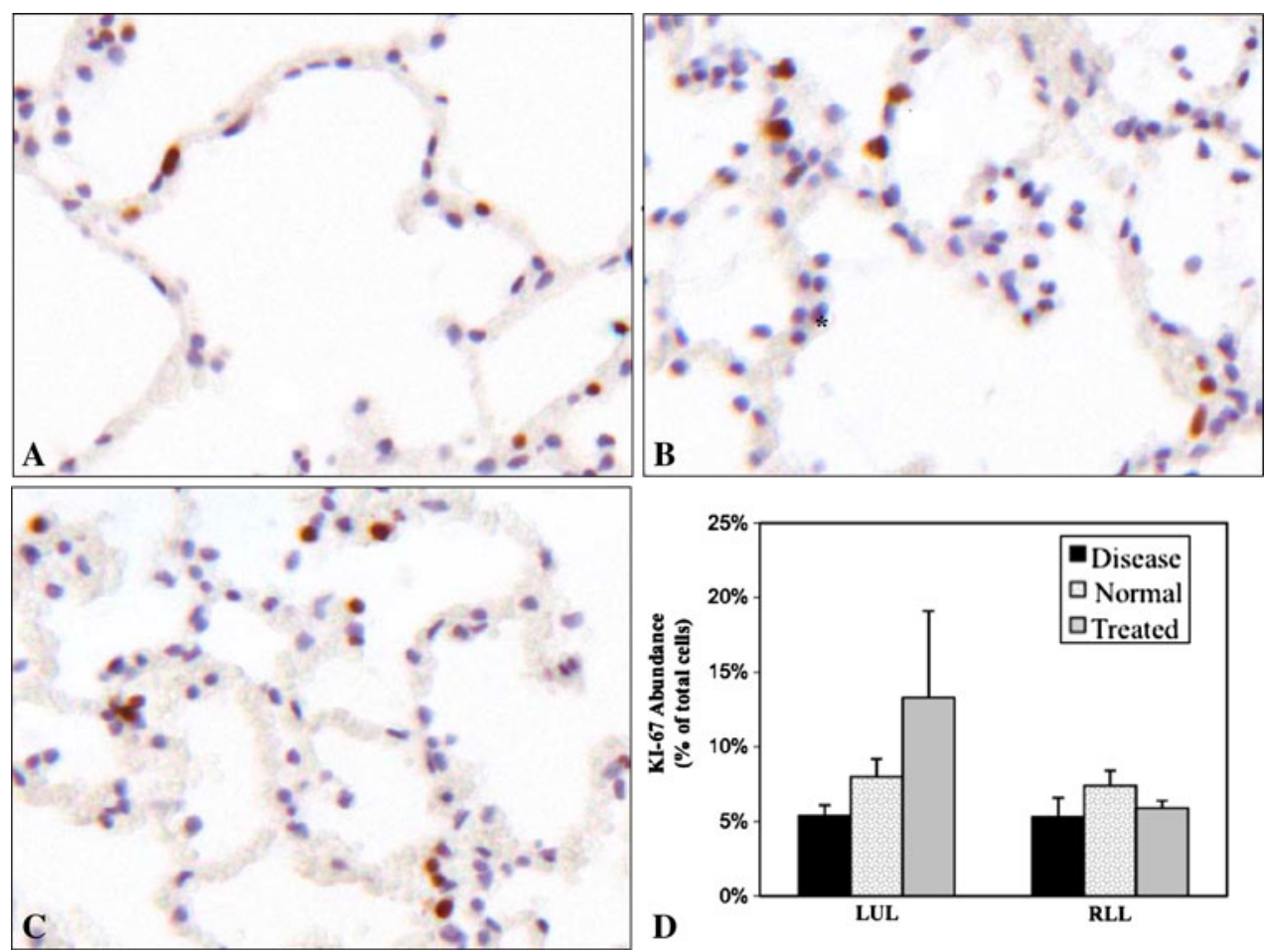

an increased number of KI-67-positive proliferating cells (LUL: $\mathrm{F}=3.04, \mathrm{p}=0.16 ; \mathrm{RLL}: \mathrm{F}=1.39, \mathrm{p}=0.33$ ) (Fig. 4) for both the LUL and the RLL. This is most likely the result of the study being underpowered.

Qualitative evaluation of histologic sections showed emphysematous dilated alveoli and an apparent reduction in red blood cells suggested poor perfusion of alveolar walls. This was particularly noticeable in the LUL (Fig. 5) closest to the rib fusion but also in the left lower lobe (LLL). The alveolar morphology was more normal in appearance in the treated lungs; red blood cells were abundant throughout the thicker alveolar walls. VEGFR-2stained cells appeared less abundant in disease lungs than in treated and normal lungs. VEGFR-2-stained cells showed frequent colocalization with Type II pneumocytes, identified by TTF-1 double stains (Fig. 6). Endothelial, macrophage, and bronchial epithelial cells were also positive for VEGFR-2.

\section{Discussion}

TIS represents a novel form of postnatal pulmonary hypoplasia and restrictive respiratory disease that occurs in children with early-onset scoliosis and chest wall anomalies. Expansion thoracoplasty improves thoracic volume in children with TIS; however, how it affects the growth and development of the lung is unknown. We presume thoracoplasty modifies the growth and development of the lung, improving alveolar structure, which may ultimately improve the ability of the lungs for gas exchange. In this study, we assessed how expansion thoracoplasty affects biologic mechanisms related to (1) development of the alveolar microstructure through cell fraction analysis of proliferating cells and macrophage; and (2) capillary development and perfusion through qualitative assessment of VEGFR-2 immunohistochemistry and hematoxylin and eosin-stained sections.

Although this pilot study was noteworthy for establishing the first animal model to explore the growth and development of lungs subjected to a constricted hemithorax and its subsequent reversal by expansion thoracoplasty, there are several limitations. First, the sample size was small and the study was underpowered as a result of restrictions placed by our institutional animal review committee until feasibility of the methods was proven. Nevertheless, some important themes were observed that provide insight as to how thoracic insufficiency might affect the development of the growing lung and how expansion thoracoplasty might compensate for these changes. Second, the rabbit model did not reproduce the severe pulmonary and spinal abnormalities seen clinically in children with TIS. Even so, interesting results were found from histologic evaluation, which can inform on the consequence of thoracic deformity on pulmonary development in clinical cases. Third, the lack of accelerated lung growth and reduction in alveolar density after expansion thoracoplasty may reflect surgical trauma (pneumothorax, 
Fig. 5A-F Representative histologic images of the left upper lobe (LUL) are shown for each experimental group (stain, hematoxylin and eosin). (A-C) Widefield images (original magnification, x200) display the relatively large airspaces in the (A) disease lung compared with the (B) treated and (C) normal lung. (D-F) Higher magnification (original magnification, $\mathrm{x} 400$ ) shows relatively poorly perfused alveolar walls in the (D) disease lung compared with the $(\mathbf{E})$ treated and (F) normal lung (red blood cells are bright pink). (E) Treated lung tissue shows enhanced perfusion and smaller alveolar spaces.

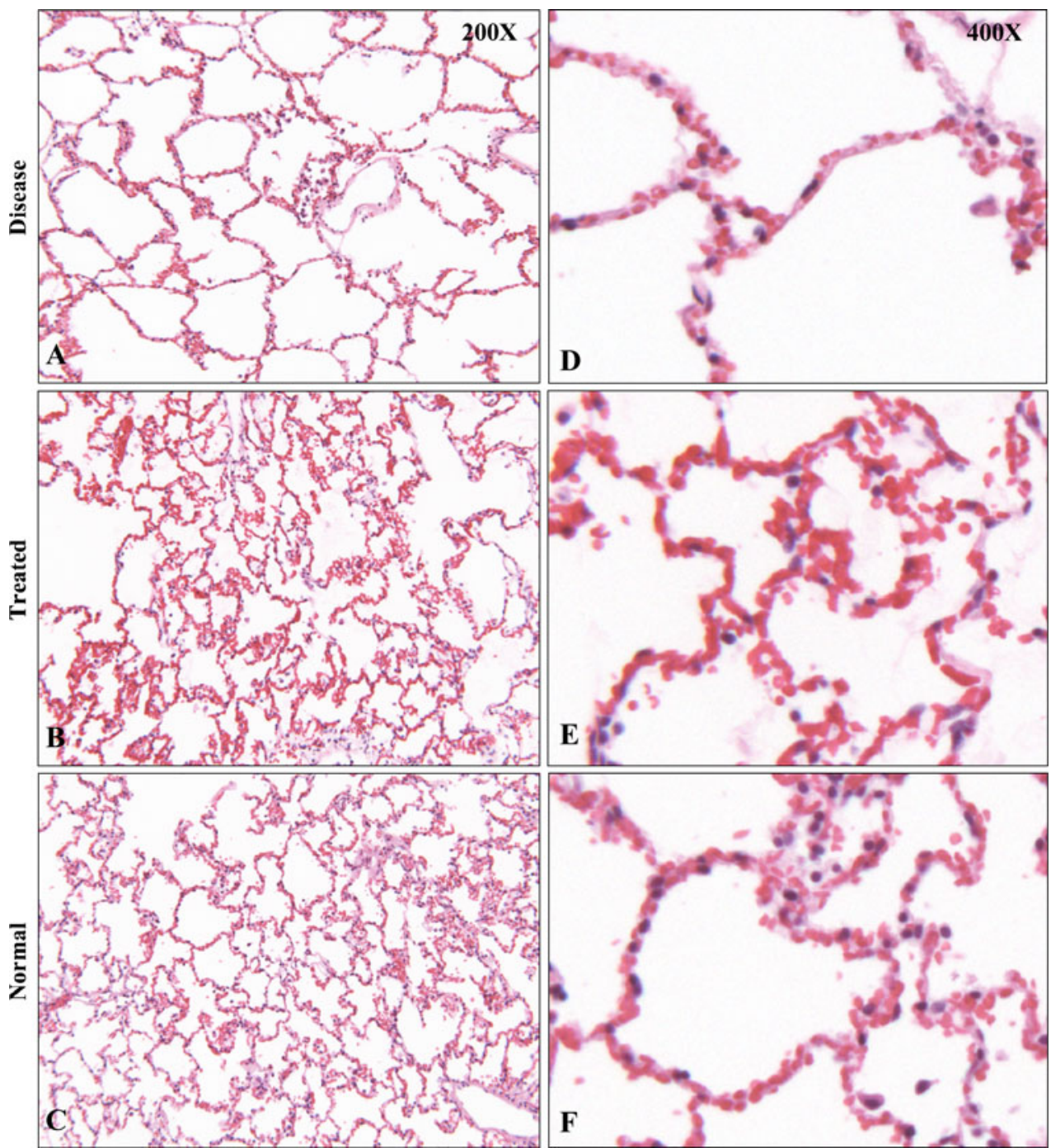

pleural scarring). We used the right lower lobe as a control rather than a sham surgery control group to identify the degree of iatrogenic effects. This control has the advantage of controlling for interanimal differences but has the disadvantage of not being from a sham surgery control animal. Last, although immunohistochemistry provided useful insights as to the factors contributing to lung development, the technique is essentially qualitative; quantitative expression assays may have been more informative.

In our current evaluation, we expected a reduced proliferating cell fraction would co-occur with the emphysematous alveolar structure and increased airspace fraction [13] found in disease rabbit lungs. A reduction in KI-67-immunopositive proliferating cells is characteristic of hypoplastic lung tissue [18]. Conversely, an increased proliferating cell fraction in treated rabbits would imply active remodeling of the alveolar morphology, including growth of the capillary network. However, our study is inconclusive on these points, most likely as a result of small sample sizes. These changes would improve respiratory function in rabbits treated with expansion thoracoplasty by facilitating $\mathrm{CO}_{2}-\mathrm{O}_{2}$ gas exchange. Similar respiratory compensation through alveolar remodeling has been observed in postpneumonectomy animal models [10, 17]. Measurements of alveolar macrophage abundance had high variance and no conclusion on relative abundance between experimental groups can be made. Macrophages phagocytize cellular debris and might have an excess presence in disease lungs as a result of infection or necrotic tissue.

Cellular signaling through the VEGF pathway [20] is a positive regulator of angiogenesis and capillary formation and an important precursor to the alveolarization phase of lung development $[5,6,11]$. We speculate an increase in capillary development and VEGFR-2 [16] abundance is present after expansion thoracoplasty; however, our immunohistochemistry results are not quantitative. 

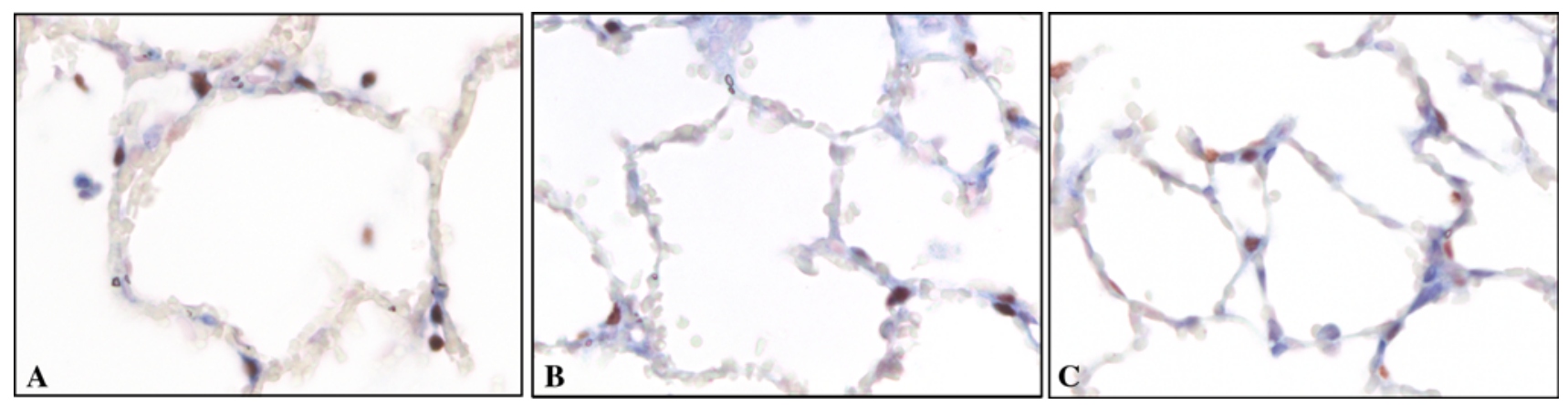

Fig. 6A-C Images show immunohistochemistry double stains for Vertical Expandable Prosthetic Titanium Rib 2 (VEGFR-2) (blue, cell surface) and thyroid transcription factor 1 (TTF-1) (red, nuclear) in the lung tissue of the three study groups (original magnification, $\times 200$ ):
(A) disease lung, (B) normal lung, and (C) treated lung. VEGFR-2 often colocalized with TTF-1-stained cells (Type II pneumocytes). In the left upper lobe (LUL), VEGFR-2-stained cells appeared less abundant in disease lungs than in treated and normal lungs.
The increased presence of red blood cells observed in hematoxylin and eosin-stained sections was conspicuous in the LUL of expansion thoracoplasty-treated rabbits (compared with disease rabbits), suggesting an enhanced alveolar capillary network. Either change will enhance the capacity of the lungs for gas exchange. In experimental studies of bronchopulmonary dysplasia in rats [19], therapy with nitric oxide improved the structure of the lung by inducing vasodilatation and enhancing pulmonary perfusion. Conversely, a long-term clinical followup study of adults with pulmonary hypoplasia induced by earlyonset scoliosis [9] demonstrated a reduction in the capacity for pulmonary gas exchange. Therefore, the enhanced perfusion observed in rabbits treated with expansion thoracoplasty may signal the initiation of alveolar remodeling.

It is currently unknown whether expansion thoracoplasty improves respiratory function and/or respiratory growth and by what mechanisms. Despite its limitations, this study suggests further hypotheses that should be tested in a future statistically powered study. Such a study will be vital to answer questions on how postnatal timing of intervention with expansion thoracoplasty affects the mechanism of long-term pulmonary development (alveolarization). Clinical pulmonary biopsies are difficult to obtain from pediatric patients with TIS and suitable control groups are unavailable; therefore, this model has great value in the study of TIS and expansion thoracoplasty intervention.

Acknowledgments We thank Melissa J. Hayward and Stephen R. Baldassarri for their assistance performing histomorphometry.

Open Access This article is distributed under the terms of the Creative Commons Attribution Noncommercial License which permits any noncommercial use, distribution, and reproduction in any medium, provided the original author(s) and source are credited.

\section{References}

1. Campbell RM Jr, Smith MD. Thoracic insufficiency syndrome and exotic scoliosis. J Bone Joint Surg Am. 2007;89(Suppl 1): $108-122$.

2. Campbell RM Jr, Smith MD, Hell-Vocke AK. Expansion thoracoplasty: the surgical technique of opening-wedge thoracostomy. Surgical technique. J Bone Joint Surg Am 2004;86(Suppl 1): 51-64.

3. Campbell RM Jr, Smith MD, Mayes TC, Mangos JA, WilleyCourand DB, Kose N, Pinero RF, Alder ME, Duong HL, Surber JL. The characteristics of thoracic insufficiency syndrome associated with fused ribs and congenital scoliosis. J Bone Joint Surg Am. 2003;85:399-408.

4. Campbell RM Jr, Smith MD, Mayes TC, Mangos JA, WilleyCourand DB, Kose N, Pinero RF, Alder ME, Duong HL, Surber JL. The effect of opening wedge thoracostomy on thoracic insufficiency syndrome associated with fused ribs and congenital scoliosis. J Bone Joint Surg Am. 2004;86:1659-1674.

5. Chang R, Andreoli S, Ng YS, Truong T, Smith SR, Wilson J, D'Amore PA. VEGF expression is downregulated in nitrofeninduced congenital diaphragmatic hernia. J Pediatr Surg. 2004; 39:825-828.

6. Dunnill MS. Postnatal growth of the lung. Thorax. 1962;17:329333.

7. Dunnill MS. Quantitative methods in the study of the pulmonary pathology. Thorax. 1962;17:320-328.

8. Emans JB, Caubet JF, Ordonez CL, Lee EY, Ciarlo M. The treatment of spine and chest wall deformities with fused ribs by expansion thoracostomy and insertion of vertical expandable prosthetic titanium rib: growth of thoracic spine and improvement of lung volumes. Spine. 2005;30:S58-S68.

9. Goldberg CJ, Gillic I, Connaughton O, Moore DP, Fogarty EE, Canny GJ, Dowling FE. Respiratory function and cosmesis at maturity in infantile-onset scoliosis. Spine (Phila Pa 1976). 2003; 28:2397-2406.

10. Hsia CC. Signals and mechanisms of compensatory lung growth. J Appl Physiol. 2004;97:1992-1998.

11. Jakkula M, Le Cras TD, Gebb S, Hirth KP, Tuder RM, Voelkel $\mathrm{NF}$, Abman SH. Inhibition of angiogenesis decreases alveolarization in the developing rat lung. Am J Physiol Lung Cell Mol Physiol. 2000;279:L600-L607.

12. Kovar J, Sly PD, Willet KE. Postnatal alveolar development of the rabbit. J Appl Physiol. 2002;93:629-635. 
13. Mehta HP, Snyder BD, Baldassarri SR, Hayward MJ, Giuffrida MJ, Entezari V, Jackson AC. Expansion thoracoplasty improves respiratory function in a rabbit model of postnatal pulmonary hypoplasia: a pilot study. Spine (Phila Pa 1976). 2010;35:153161.

14. Mehta HP, Snyder BD, Callender NN, Bellardine CL, Jackson AC. The reciprocal relationship between thoracic and spinal deformity and its effect on pulmonary function in a rabbit model: a pilot study. Spine. 2006;31:2654-2664.

15. Ruifrok AC, Johnston DA. Quantification of histochemical staining by color deconvolution. Anal Quant Cytol Histol. 2001; 23:291-299.

16. Shen BQ, Lee DY, Gerber HP, Keyt BA, Ferrara N, Zioncheck TF. Homologous up-regulation of KDR/Flk-1 receptor expression by vascular endothelial growth factor in vitro. J Biol Chem. 1998;273:29979-29985.

17. Takeda S, Hsia CC, Wagner E, Ramanathan M, Estrera AS, Weibel ER. Compensatory alveolar growth normalizes gas-exchange function in immature dogs after pneumonectomy. J Appl Physiol. 1999;86:1301-1310.

18. Thomas PA, Durbin J, Langston C, Raab SS, Jagirdar J, Greco MA. Growth fraction determination in pulmonary hypoplasia using Ki-67 (MIB-1) antibody. Pediatr Pathol Lab Med. 1996;16: $745-753$.

19. Tourneux P, Markham N, Seedorf G, Balasubramaniam V, Abman SH. Inhaled nitric oxide improves lung structure and pulmonary hypertension in a model of bleomycin-induced bronchopulmonary dysplasia in neonatal rats. Am J Physiol Lung Cell Mol Physiol. 2009;297:L1103-L1111.

20. Voelkel NF, Vandivier RW, Tuder RM. Vascular endothelial growth factor in the lung. Am J Physiol Lung Cell Mol Physiol. 2006;290:L209-L221.

21. Weibel ER, Gomez DM. Architecture of the human lung. Use of quantitative methods establishes fundamental relations between size and number of lung structures. Science. 1962;137:577585 . 